All prospectively identified patients were treated with a second course of IV acyclovir, and 4 received immunotherapy with improvement in 3.

\title{
References.
}

1. Armangue T, et al. Ann Neurol. 2014 Feb;75(2):317-23.

\section{CONTINUOUS EEG IN ENCEPHALITIS}

Investigators from Rady Children's Hospital of San Diego, CA, reviewed records from all 217 children enrolled in the California Encephalitis Project 2004-2011. At least one seizure was observed clinically or recorded on EEG in $100(46 \%)$ children. Diffuse slowing occurred in $88.9 \%$, focal abnormalities in $63.2 \%$, and epileptiform abnormalities in $57.3 \%$. Continuous EEG for at least 1 day in $54(25 \%)$ patients recorded a seizure in more than half of patients. In $22(10 \%)$ children, a seizure was recorded by continuous EEG after routine EEG had failed to record a seizure. Overall, a continuous EEG was more likely to capture a seizure, capture a subclinical seizure, or rule out an event as a seizure than routine EEG ( $<<0.0001$ ). (Gold JJ, Crawford JR, Glaser C, Sheriff H, Wang $\mathrm{S}$, Nespeca $\mathrm{M}$. The role of continuous electroencephalography in childhood encephalitis. Pediatr Neurol 2014 Apr;50(4):318-23).

COMMENTARY. Continuous EEG is an important diagnostic tool in the ICU, to identify nonconvulsive seizures or status epilepticus, aid in management of AED treatment, and to identify seizures in children with suspected encephalitis. An online survey of pediatric neurologists from 50 US and 11 Canadian institutions conducted in 2011 found the following common cEEG indications: altered mental status after status epilepticus (97\%), altered mental status of unknown cause $(88 \%)$. Median number of patients with cEEG per month per center increased from 6 per month in 2010 to 10 per month in 2011 in the US [1].

\section{References.}

1. Sanchez SM, et al. J Clin Neurophysiol. 2013 Apr;30(2):156-60.

\section{BRAIN TUMORS}

\section{COGNITIVE OUTCOME OF CRANIOPHARYNGIOMA}

Investigators from Carl von Ossietzky University, Oldenburg, and other centers in Germany, compared cognitive performance in a group of 15 patients with childhood craniopharyngioma and hypothalamic involvement and a group of 24 age- and intelligence-matched control subjects. IQ scores were mostly in the average range and not significantly different in patients and controls. Patients had significantly lower performance scores in tests of memory and executive functioning. Delayed recall performance was severely impaired in one-third of the patients. Compared with patients with low-grade hypothalamic involvement, those with high-grade involvement showed worse performance in executive functions and reduced functional aptitude for daily life actions. Preoperatively, only 1 patient was severely obese; postoperatively, most patients 
were obese, a sign of hypothalamic dysfunction. (Ozyurt J, Thiel CM, Lorenzen A, et al. Neuropsychological outcome in patients with childhood craniopharyngioma and hypothalamic involvement. J Pediatr 2014 Apr;164(4):876-881).

COMMENTARY. Craniopharyngioma, a histologically benign tumor, may invade the hypothalamus, mammillary bodies, pituitary, and optic nerves. Sequelae of the tumor or its removal include visual field defects, obesity, and neurobehavioral deficits. Hypothalamic obesity in $46 \%$ of 24 cases of craniopharyngioma treated at the Phoenix Children's Hospital was refractory to current management options and accounted for increased mortality [1]. The development of obesity is influenced by premorbid obesity, genetics, and therapy received, especially radiation.

\section{References.}

1. Rosenfeld A, Arrington D, Miller J, et al. Pediatr Neurol 2014 Jan;50(1):4-10.

\section{RISK OF FAMILIAL INTRACRANIAL ANEURYSM}

Investigators at University Medical Center Utrecht, Netherlands, studied the yield of long-term (up to 20 years) screening for intracranial aneurysms in individuals with a positive family history ( 2 or more first-degree relatives) of aneurysmal subarachnoid hemorrhage (aSAH) or unruptured intracranial aneurysm (1993-2013). MRI or CT was performed from age 16-18 to 65-70 years. Aneurysms were identified in $51(11 \%)$ of 458 individuals at first screening, in $21(8 \%)$ of 261 at second screening, in $7(5 \%)$ of 128 at third screening, and $3(5 \%)$ of 63 at fourth screening. Five (3\%) individuals with 2 negative screens had a de-novo aneurysm in follow-up screens. Smoking, history of previous aneurysm, and familial history of aneurysms were significant risk factors for aneurysms at first screening. History of previous aneurysms was the only significant risk factor for aneurysms at follow-up screening. Aneurysms were identified in 6 (5\%) of 129 individuals screened before age 30 years. Long-term serial screening is advocated in individuals with a family history of aSAH. (Bor ASE, Rinkel GJE, van Norden J, Wermer MJH. Long-term, serial screening for intracranial aneurysms in individuals with a family history of aneurysmal subarachnoid hemorrhage: a cohort study. Lancet Neurol 2014 Apr;13(4):385-92).

COMMENTARY. Of 77 children (mean age 12 years) with 103 intracranial aneurysms treated at University California San Francisco (1981 and 2008), 25 (32\%) presented with subarachnoid hemorrhage. The aneurysms were saccular in $35(45 \%)$, fusiform in 25, traumatic in 12 patients, and infectious in 6 . Treatment of 59 patients was conservative in 18 . Mortality was $1.3 \%$ [1].

In a long-term study of 114 pediatric patients with aneurysms at Helsinki University, Finland, the mean patient age was 14.5 years (range 3 months to 18 years) and the male:female ratio was $3: 2$. The most common location was the internal carotid artery bifurcation (28\%). A family history of aneurysms was present in $14(12 \%)$ [2].

\section{References.}

1. Hetts SW, et al. AJNR Am J Neuroradiol. 2009 Aug;30(7):1315-24.

2. Koroknay-Pal P, et al. J Neurosurg Pediatr. 2012 Jun;9(6):636-45. 